\title{
Günter Frankenberg Demonstrationsfreiheit - eine verfassungsrechtliche Skizze
}

\author{
1. Demonstrationsfreibeit - eine staatiche Konzession, ein Privileg oder eine \\ konstitutive Garantie demakratischer Prozesse?
}

Das Grundgesetz nimms die Freiheic zu demonstrieren, ebenso wic das Surcikrecht, nicht wörtlich in Schutz. Dennoch, und darüber berrscht nahezu Konsens, kommt dieser Freiheit der Status einer verfassungsmäßigen Verbürgerung zu.' Welcher grundgeseczlichen Freiheitsgaranrie das Demonstrationsrecht allerdings zu entmehmen isc, bleibc unentsclieden. Die Mehrzahl der Kommentatoren und dic Judikatur neigen dazu, politische Demonstrationen als Unterfall der Versammlung unter freiem Himmel oder als Aufzug im Sinne des Versammlungsgesetzes dem Ar. 8 GG zu subsumicren. ${ }^{2}$ Zugleich oder abweichend davon gelten sie aber aucli als kollektive Meinungsäußerungen oder als Informationsquellen im Sinne des Are. S I GG'; als wixtum compositum im Einzugsbercich von Meinungs-, Versammlungs- und Vereinigungsfreiheit; ; als von Art. 2 I GG geschützte Handlungen - aul Selbsiverwirklichung oder darauf angelegt, dic Isolacion des Individuums aufzuheben'; schließlich als punktuelle Plebiszice oder Formen polirischer Willensbildung, deren Legitimitï sich aus dem Demokraticprinzip ergibr? Auf den ersten Blick mag dic Zuordnung der Demonstrationsfreiheit zu diesem oder jenem Grundgesetzartikel wie ein müßiges, weil praktisch folgenloses dogmatisches Verwirrspiel erscheinen, das nichts als dic Mobilisierbarkeit rechtlicher Argumente für beliebige Auffassungen und Zwecke unter Bereis stell. Zuordnungen enthalten jedoch eine Vorentscheidung über die legitimen Funktionen von Demonstrationen und über die Schranken demonstrativen Protests und plebiszitärer Willensbildungsprozesse. In den Funkrions- und Schrankencrwägungen scheinen unterschiedliche verfassungstheorerische Konzeprionen auf, die durchaus handgreifliche Folgen haben für die Inanspruchnahme der Demonstrarionsfreiheit.

Nach einhelliger, vorwiegend am liberaien Freiheitsverständnis oricntierter Auffassung hat die Freibeit zu demonstrieren (zumindest auch, wenn nichı gar primär) den

1 Herzog, an: Maunz-Dürig-Herzog (MDH), GG, Ar. 8, RdNr. 1 ff.; S. Ou, Das Reche auf freie Demonstration (Neuwied/Berlin, 2967) $\mathrm{m}$. w. Nachw. Samper lehnt dagegen den Verfassungsrang der Demonstrationsfreihcit ab und operier - insowes folgerschrig - mit Anfülirungszeichen: Recheslragen zum .Demonseracionsrecht $a$, Bay VBI. 69, $77 f i$.

2 : Münch, GG, Ar. 8, RdNr. 9; vgl. v. Mangoldi-Klein, Das Bonner GG, 2. Aufl, Art. 8, Anm, Il 2 a und 111: Ou, Gesetz uber Vessammlungen und Aufzuge, 3. Aufl. (Stulgary/München/Hannover, 1979), 24.

3. Vgl. Ou, Geserz .... A, 2. O, 37 und Ossenbuhi, Vers.mmlungs/reihcit und Spontandemonstration, in: Der Staas 1971, s3 ff., 6o 1 .

4 Zeidlcr, AuBerparlamenearische Bmvegungen, Demonsualionsroche und Widerseznd, in: Das Rechr auf Dimonstration (1969), iff., 6.

s Herzog, MDH, An. 8, RdNr

6 F. Ossenbühl, a. 2. O., 64 .

7 Vgl. E. Denninger, Demonstraxionsfreilzeil und Polizeigewalt, ZRP 68, 2 ff.; ders. Thesen zur Demonstrationsfrcihelt, DRiZ 69, 7I ff; Otl. Demonsemtionsredu und Meinungsírcihere, DRiZ 69. $66 \mathrm{ff}$; H. Hannover, Demonsemeionsfreiheit als demokrasisches Grundreche, KJ $6 \$$, if If. 
Charakter eines negativen Stacusreches, eincs "Abwehrrechrs", das die Sphäre staatsbürgerlicher A ktivität teilweise von stâtlicher Ingerenz freisetzt, zugleich aber auch jede Zwangsverpflichtung zur Teilnahme an hohcitlich angeordneten Akklamationsveranstaliungen und damit zur öffentlichen Äußerung einer politischen Gesinnung untersagt. ${ }^{8}$ Soweit in diesem Rahmen Demonstrationen mit Auf- und Umzügen aller Art gleichgeserzt und als Sondernutzung der öffentlichen Wege und Plätze gewürdigt werden, ergeben sich hinsichtlich der Einschränkbarkeit zwei Alternativen: Encweder untersteht dic Demonstrationsfreiheit einem allgemeinen Gemeinwohl- und Ordnungsvorbehalt, den die Polizei auf der Grundlage der polizeirechclichen Generalklausel exekutiert", die sie befugc, alle für dic Unterbindung und Bescitigung s'on Störungen erforderlichen Maßnahmen zu treffen. Oder aber Demonstrationen gelten wie Versammlungen unter freiem Himmel als apolizeifesr $\propto$ mit der Folge, daß einschränkende staatliche Maßnahmen einer spezialgesetzlichen Ermächtigung bedüríen. ${ }^{\circ}$ Beide Varianten enckleiden freilich Demonstrationen ihres spezifisch politischen Charakters als Protestform und privilegieren de facto - wie auch das VcrsammlG" - kollekrive Aktionen, die sich nicht als Beiträge zur öffentlichen Diskussion verstehen, sondern die, wie etwa Volksfeste, Wallfahren oder Prozessionen, das Bedürfnis nach Zerscrcuung befriedigen oder kulturell-religiösen Traditionen und Manifestationen Ausdruck geben sollen. Verfassungstheoretisch betrachter ist damit im sensiblen Bercich der Freiheit politischer Kommunikation entweder die Entscheidungsfreiheit der Exekutivbehörden oder die in Zweifel nur durch die "Schranken-Schranken a", insbesondere die Wesensgehaltsgarantie des Art. 19 Abs. 2 GG, limitierte Regelungsbefugnis des Geseczgebers installiert, d. h. die Arrikulationschancen ron Minderheiten stehen zur Disposition jeweiligen Parlamentsmehrheit.

Das ndemokratisierte liberale Freiheitsverständnis erkennt demgegcnüber dic Demonstrationsfreiheit auch als aktives Statusyeche an. "s Sic ist demnach "Mittel zur Teilnahme am policischen Willensbildungsprozeßa". Wie der Wahlakt geiten Demonstracionen als Versuch, die staatliche Willensbildung zu beeinflussen. "Diese Konzeption betonc den Einfluß-und Teilhabecharakrer der Demonstrationsfreiheit und schreibc zugleich die liberale Tradition fort, indem sie diese Garancie primär unter dern funktionalen Aspekt ihrer spezifischen Leistungen zur Stabilisierung des politischen Systems betrachter. Zwar verweisen die Begründungen auf den demo-

8 Zum status negatwus exemplarisch Her7.og, MDH. An. 8, RdNr. 34 ff.

9 G. Dürig, AoR 79 (195;/S4), 80; 1. A. v. Mangoldt.Klein. An. 8, Anm. IV 4. Zur Demonstration als Sondernutzung vgl. Wollf-Bachof VenvR I, \$ 99 IJ: Dietel-Ginczcl. Demonstrations- und Versammlungstreiheit, Komm. zum Cesclx i. Versammlungen und Aufzuge, 6. Aufl. (Köln, 1979), $\ 1$, Anm, 17.

10 Wie sic im VersammlG und Bannmeilen $G$ enthalten sind. $V_{g l}$. Dietel-Gintzel, \$ 1, Anm. 17. Allstings cnehälı $\$$ is I VersammlG selbse eine generalklasclaruge Formulienng, die jedoch reseriktiv auszulegen ist, v. Müncl, Art. 8, RdNr. 34; Herzog, MDH, An. 8, RóNr. 99. Zum Práventiverbot bej uniriedlichen Versammlungen vgl. v. Münch, Art. 8, RdNr. 26.

1I $\$ 17$ VersammlG dispensiert »Gottesdienste uneer freiem Himmel, Bungünge und Walliahreen. getvöhnliche Lacichenbegräbnisse, Züge von Hochxciesgcsellschafien und hergebrachte Volksfeste $*$ von den einschneidenden Einschränkungen des VersammlG; vgl, xur Kritk E. Denonger, Demonstrationsfreiheit...., a. a. O., 42 und Ou, DRiZ $69,67$.

12 A ußer der Wesensgehaltsgarantic hat vor allem der Grundsarz der Verhältnismäßigkeit die Bedeutung einer Schranke für einschränkende staatliche Maßßnahmen; vgl. D. Mercen, Gedanken zur Demonsera. rionsfreiheit, MDR 68,621 ff., 623 .

1) BVerfGE 7, 210: 9,68 und 1 12; 20, 99. Dictel-Ginixcl, \$1, Anm. $18 \mathrm{~m}$. w. Nachw.

14 Dietel-Ginizel, 1 , Anm. 18; Ou, Das Recht auf freic Demonstration, 1. a O., 30.

is D. Merten, Demonstrationen unter dem Gesetx, Schriften der Bundeszentrale 1. polit. Bildung (Bunr, 1969), ss ff., s6. H. H. Kleia, Zur Auslegung des Rechesbegriffs der woffenelichen Sicherheir und Ordnung*. DVBI. 71,233 . 
kratischen Kontext; allerdings zeichnen sie die mittelbare, repräsentativ-parlamentarisch strukturierte Dcmokrarie als (einzig) grundgesetzkonform und praktikabel aus, die politischer Demonstrationen nur als Korrekriv oder Komplement bedarf. "* Daß die Freiheit, äffentlich zu demonstricren, als akrives Statusreche einen besonderen Rang hat, weil es eines der Grundrechte ist, die für die Demokratie "konsciturive Bedeutung “ habcn'7, weil cs ferner nauch unterprivilegiercen und von Organisationen oder Massenmedien nichr gctragenen Gruppen (ermöglicht), auf ihre Ansichten aufmerksam zu machen ${ }^{\prime s}$, weil es schließlich sMimcl zur Realisierung der Volkssouveränitä durch Partiziparion «' ist, bleiben Einsichten, die durchweg keine systematischen verfassungsrechtlichen und demokratietheoretischen Folgen haben. Am Gesetzesvorbehalt dieser Frciheirsgaranzie wird richr gerühr. Damir bleibr die Delinitionsgewalı über die Zulässigkeit und Unzulässigkeit politischen Protests nach wic vor in den Händen der parlamencarischen Mehrheit, deren Entscheidung freilich ciner verfassungsgerichtlichen Überprüfung standhalten muß.

Keine Option für das etablierre politische Inscitutionensystem auf Kosten außerparlamentarischer Willensbildungs- und Oppositionsformen enthält demgegenüber cin Begründungsansatz, der dic Demonstrationsfreileeit als demokratisches Grundrecht ${ }^{20}$ oder als Element des status constituens" begreift. Demonstrieren heißt hier nicht mehr nur, am staatlich organisierten Willensbildungsprozeß bestenfails im Sinne korrigierender politischer Einflußnahme teilzuhaben; vielmehr wird nun die eigene politische Becätigungsfreiheit der Bürger in den Mitcelpunkt gerückt. Deren konstritucionelle Verankerung soll einmal erlauben, durcl öffencliche Diskussion und kollekrive Akrionen jedc offizielle Bcwußrseinsmanipulation zu durchbrechen und über Machtverhältnisse in Scaar und Gesellschaft aufzuklären." Demonstrationen fungieren gleichsam als öffentliche Herrschafts- und Idcologiekritik. Oder aber sie sollen den "Staa überhaupt erst (mirerzeugen)a und werden dcshalb konsequentcrweise vor staatlichen Einscliänkungen bewahrt, solange sic nicht in *revolutionáre" A ktionen umschlagen, die bei der gewaltsamen Durchsetzung politischer Ziele normative Schranken hinsichelicl der eingesetzten Mittel nicht kennen. ${ }^{25}$ Nicht abstrakte Rechrsgüter, wie die öffeneliche Sicherheit und Orónung, sondern die handgreifliche Bedrohung konkreter Rechrsgüter, wie insbesondere die Rechee anderer Individuen auf körperliche Unversehrtheit, persönliche Ehre und eine geschüzre Privatsphäre markieren demnach dic Grenze der Demonstrationsfreiheir. Ein unbestreitbarer Vorzug dicser Auffassung ist, daR sie den Parlamentsvorbehalt zurückdränge und den strafrechrlichen Rechtsgürerschurz ins Blickfeld rückr. Eine Konkrecisierung der Freilacirsgrenzen steht jedoch nach aus. ${ }^{4}$

$16 \mathrm{Vgl}$ Ossenbuht, a. a. 0., 62, der aus dem statks aciisus die Unzulässigkeit einer inhalelichen Bewerung von Demonsirationszitelen folgert.

17 ч. Münch, GG, Ar. \&, RdNr, a sieht in Demonstrationen - Zeichen policischen Engagements in völlıger Öfentichkcir " und bewertet sie grundsätz.lich positiv. Als Grundreche habe dıe Demonstrationstreileit lur die Demokratie xkonstilutive Bedeutung*.

18 v. Munch, GG, Art. 8, RdNr. 2.

19 Dietel-Gintzel, $\$ 1$, Anm. 21 .

20 H. Hannover, a. a. O. (Anm. 7).

21 E. Denninger, Polizei in der freiheitlichen Demokratie (Franklure/Berlin, 1968), if fl.; ders., Demonstrationsfreilyeit und Polizeigewah, a. a. O. Zur Kritik dieser Auffissung vgl. Ossenbülıl, 2. $2,0 ., 626$.

22 H. Hannover, a. a. O., 53 f

2) E. Denninger, Demonstrationsfrciheit und Polizcigcwalt, a. 2. O., 44.

24 Denninger, a. a. O., \{3 I, zicht die Grenze zwischen Reform und Revolurion, zwischen zulassigem und unzulässigem Protest \%u Rtcht nicht automatisch entlang der Legalititslinie, sondern verlagert sıc zunachst ins Bewustscin der theoretisierenden und agierenden Demonstranten. Damit verweist er dic Polizci zwar nicht unmittclbar auf eine ungewisse Motivforschung, wohl aber aul einc (durch cine 
Nimmt man die beiden zuletzt skizzierren, eine Mindermeinung repräsentierenden Ansätze aus, so sind besonders zwei Merkmale für den verfassungsjuriscischen Diskurs über Inhalt und Umfang der Demonscrationsfreiheic kennzcichnend: Da diese nicht systematisch im Kontext einer demokratischen Verfassung und deshalb als Garantie politischer Kontroverse und Bewußtscinsbildung begriffen wird, hat sie tendenziell den Starus ciner Freiheitsgarantie 2. Klasse, die der cinfachgeserzlichen Domestizicrung zugänglich ist. $\mathrm{Da}$ Demonstrationen außerdem als kollektive Adressen von unzufriedenen Bürgern wahrgenommen werden, die wie Pecitionen an scaatliche lnstanzen und politische Repräsentanten zu richten sind, als Kommunikation zwischen Regierenden und Regierten also, werden ihre politischen Funktionen typischertveise aus der Perspektive des politischen Systems bestimmt, nicht aber als originäre Artikulation des souveränen Bürgerwillens verstanden. Eine prinzipiengeleitete demokrarieangemessene Begründung des Demonstrationsrechts ist daher nur in Ansätzen entrvickelt worden. Dieses Defrzit nehmen wir zum Anlaß, ausgehend von den fundamencalen Organisacionsprinzipien des Grundgesetzes, wenigsens die Umrisse eines verfassungsthcoretischen Bezugsrahmens für dic Demonstrationsfreiheit zu skizzieren und die verfassungsrechtichen Vorgaben zu identifizieren - mit der Absicht, die demokratickonforme Demonstrationspraxis nicht nur "polizei- , sondern auch "parlamentfests zu machen, $d$.h. vor allem dem Zugriff des Strafgesetzgebers zu entziehen.

\section{Volkssouveränität und Minderbeitenschutz als konstilutive Prinzipien demokratischer Selbstregicrung}

Bezugspunkı jeder öffentlich propagierten, an ein ziviles Publikum oder an staacliche Instanzen gerichteten Meinung, Kritik oder Fordenung ist im weitesten Sinne der Prozeß, in dem über die ngemeinsamen Geschäfre der Gesellschafc (auch wenn sie nur partikulare Gruppen becrelfen) beraten und entschieden wird. Die Inhalts- und Grenzbestimmung des Demonstrationsrechts hat sich daher primär an den für diesen Prozeß konstituciven normativen Leitmarken, an den staatsorganisatorischen Grundsätzen zu oriencieren. Was »konstituriv o oder "fundamental « ist, darüber gibt es üblicherweise Disscns. Das Grundgesecz - in diesem Punkte ungewöhnlich entschieden - läßr wenig Dissens zu. Jedenfalls die in Art. 79 III GG für veränderungsfest erklärten Organisationsprinzipien des Prozesses politischer Willensbildung dürfen wir als "fundamental a auszeichnen: die vom souvcränen Volk getragene, rechisstaatich-grundrechclich gebundenc, gervaltenteilige Ausübung politischer Herrschaft im Gesamtrahmen einer demokratischen Staatsform.

Dic deutsche Staats- und Verlassungslchre hat sich cbenso wie die Veríassungsrechtsprechung für den Gedanken der Volkssouveränität nie sonderlich erwärmen können"s und stets dazu geneigt, den staatlichen Organen eine Souveränitärsprämie

emsprechende Ausbildung zu erverbende) "Kunst der Prognose und dic Beachtung des Grundsatzes der Verhälenismäßigkeil. Sowohl solehe Prognosen als auch Verhälınismäßigkciesenvägungen bedürfen jedoch prinzipiellet verfassungsrechtlicher Orıentierungen, soll niche die Tendenz bürokratisch organisicrter Sicherheitsapparate und dic Neigung ibrer Vollzugsorgane, allenthalben srevolutionäre Innentendenzen - zu vernuten, wo nur demokratische Auseinandersetzungen vorliegen, am Ende doch vieder durchschlagen.

2s Im Verhälenis zu den weitreichenden demokracie- und verfassungstheoretischen Implikntionen und ru den politisch-praktischen Konsequenzen ise die cinschlagige neuere Literacur uberschaubar geblieben; vgl. vor allem die Beiträge in H. Kurz (Hg.), Volkssouverinität und Stătssouveränität (Darmșadt, 1970): P. Graf Kielmannsegg, Volkssouverünicat (ra77); D. Schefold, Volkssouveränität und repräsentative Demokratie in der schweizerischen Regeneration 1830-1848 (Basel/Stungart, 1966) und zur Diskussion um den Souveränitätsbegriff P. Häberle, AoR $92(1967), 299$ if. 
zu geben. ${ }^{26}$ Die sciefmützerliche Behandlung mag auf den Beigeschmack von Revolurion zurückzuführen sein oder darauf, daß Volkssouveränitär tradicionell auf eine Polemik gegen die Fürstensouveränitär und gegen die kacholische Souveränitärslehre, nach der alle Obrigkeit von Golt ausgehe, verkürat wurde. ${ }^{77}$ Das Grundgesetz zwingt zu einer aktuelleren Sicht; denn es legr apodiktisch in Art. 20 II If fest: «Alk Sraarsgewalt geht vom Volke ausa, Man mag diese Festlegung und insbesondere den ihr entsprechenden Satz der Präambel ("... hat das deutsche Volk ... kraft seiner verfassungsgebenden Gewalt diescs Grundgeserz . . beschlossen $\times$ ) als empirische Aussage bescreiten ${ }^{\prime 8}$, als normativc bleibr sie gültig und für die Verlassungsinterpretation verbindlich. ${ }^{29}$ Originärer Träger der Souveränitär - Staatsgewalt und verfassungsgebender Gewalt - sind demzufolge die Bürger in ihrer Gesamtheit. Die apodiktische Formulierung des Art. 20 II x GG läßı gleichrangige Legitimationsprinzipien politischer Herrschaft nicht zu. Auch Art. 20 II 2 GG gibt nichrs für dic These her, hicr werde dem Volke die eben «uerkannte Souvcränirät wieder abgegraben. Denn im Unterschied zu vielen Kommentatoren des Grundgesezzes unterscheider dieses klar zwischen Trägerschaft und Ausübung der Staatsgewalt. Ebenso wie Volkssouveränität cine logische Voraussetzung demokrarischer Selbsibestimmung und Selbstregierung ist, ist die Ausübungsregelung des Art. 20 II 2 GG deren organisalionsrechtliche Konsequenz..$^{\circ}$ Auch auf der Ebene der Ausübung der Staatsgewalt gibt das Grundgesetz den Anspruch nichr preis, daß Demokratie immer auch Selbsttütigkeit der Bürger bedeuter und sich nicht in deren Repräsentation erschöpft. Gegen die Interpretation des Art. 20 II GG aus der verengren Perspekcive dcr Funktionsgarantien und Funkrionserfordernisse eincs repräsentaciven parłamentarischen Systems ist hervorzuheben, daß das Grundgesetz die Ausübung der Staatsgewalı durch die Akrivbürger selbst und durch besonderc Organe in gleicher Weise vorsieht, also nichr die mittelbare Demokratie vor- oder festschreibr und dic Bürger im ubrigen darauf verweist, ihre Repräsentanten zu wählen und durch repräsentative Organe und Gerichte kontrollieren zu lassen." Niches anderes ergibr sich aus der Funktionsbesrimmung der Grundrechte der Art. 4, 5, 8, 9, 11 GG als politische Teilhaberechre.

Diese Auslegung muß sich auf eine Reihe von Einwänden gefaßt machen: Sie nklebe" systcmblind am Wortaur des Art. 20 II GG und verkenne, daß der Verfassungsgeber die plebiszitäre Alternative außer in Art. 29 GG nicht weiter verfolgt habe; sie gehe am Willen der Verfassungsväter und deren Einsicht in die mangelnde Reife des Volkes sowic an den praktischon Schwierigkeiten mir plebiszitären Entscheidungsformen vorbei und berücksichtige nicht die Komplexität der politischen Probleme in einer Massendemokratie, ${ }^{12}$ Im Ergebnis weisen diese Einwände, die zugleich das herrschende Demokratieverständnis umreißen, den Bürgern die Rolle zu, in erster Linie ihr Wahlrecht auszuüben, in zweitcr Linie im Medium einer politisch räsonierenden Öffentlichkeit auf die Parteien einzuwirken

\footnotetext{
$26 \mathrm{Vgl}$, dazu DuR, $4 / 80$.

$27 \mathrm{Vg}$. Wernicke, BK, Arr, 20. Anm. II 2.

28 So z. B. H. Sclnneider, NJW" 44.937 ; v. Munch, Prambel, RdNr, 17 hält die Aussage auch als empirische für richtig, $\mathrm{d}$ a sich das Volk auch durch seine Reprasentanten einc Veriassung gcben konne.

29 K. Hesse, Grundzüge des Verfassungsrechis der Bundessepublik Deuesclyand, 12. Auf). (Kartsruhc, 1980). s5: MDH, Art. 20. RdNr. $33 ; 8 \mathrm{VLrfGE}_{49}$, $89 / 124 \mathrm{f}$.

yo Vgl. v. Mangoldt-Klein. An. zo, Arm. V 4 b, s; E. und G. Küchenholl, Allgemeine Staatslchre, 2. Auil. (Köln/Stuttgant, 19:1), 119 (f., $123 \%$.

II So aber die ganz überwicesnde Auslegung dis Art, 20 I, II und der Kommunikationsfreiheiten; vgl. y.

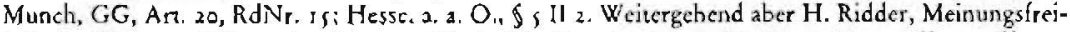
heit, in: Neumann/Nipperdey/Scheuncr (Hg), Dic Grundrechte Il (Berlin, 1968), 243 ff., 249 ff.

32 Vgl. etwa MDH, Ar. 20, RdNr. 38 ff.
} 
und im übrigen durch dic statsbezogene Inanspruchnahme ihrer Freiheitsrechte die ndurch und durch repräsentasiv strukturierte " Demokratie zu becinflussen. ${ }^{33}$

Diese Einwände sind freilich nicht stichhaltig. Erstens ist eine enge Orientierung am Verfassungstext, zumal wenn es um Grundsäıze geht, kein vorwcribarer Umgang mit dem Grundgesetz. Textnähe drückt zunächst den gebotenen Respekt vor einer schriftlichen Verfassung aus und schränkt den Spielraum der Interpreten ein, dic versucht sein könnten, konstitutionclle Garancien für die jeweils favorisierten politischen Vorurteile und Zwecke zu instrumenralisieren." Zw Zwcitens enthält die These, im Grundgesetz seien die plebiszitären Ansärze nicht weiter entlaltet, cine verdeckte grundrechtstheoretische Prämisse: Denn Demonstrationen, Versammlungen und ähnliche politische Aktionen, aber auch Streiks scheiden danach als Formen plebiszitärer Bewußeseins- und Willensbildung und damir ais verlassungsmäßig verbürgte Alternative zu den parteimäßig organisierten oder parlamentarisch institutionalisierten Willensbildungsprozessen aus. Die "punktuellen Plebisziteu, die ja sehr wohl auch der Selbstverscändigung der Bürger dienen und deshalb nicht auf eine Kommunikarion zwischen Regiercen und Regierenden reduzierbar sind, gelten der herrschenden Lehre und Rechtsprechung als bloßer Annex des parlamentarisch-repräsentariven Enrscheidungsprozesses. Dem Grundgesetz wird so eir überkommenes liberales Dcmokratiemodell und Freiheitsverständnis impuriert, in dem zivar die demokratiekonscitutive Bedeutung der Meinungsfreiheit gewürdigt wird, für die Vorstellung, Demonstrationen gehörten zum Kernbestand nichtdelegierter Souveränität, jedoch kein Raum bleibr. Die Grenze zwischen der Ausübung nichrdelegierter Souveränität und der Ausübung der Staatsgewalt durch besonderc Organe wird dadurch verwischt, daß die Betätigungsfreiheic der Bürger als "Vorformung des politischen Willens" qualifizicrt wird, die eben nur im vor-parlamentarischen Raum statufinde und auf den parlamentarischen EntscheidungsprozeB ausgerichtec sei, wenn sie nicht gar nur der individuellen Selbstentfaltung diene.

Der Überzeugungskraft und Aurhentizität dieser Interpretarion des grundigeseczlichen Demokratiemodells selbsc mißtrauend, schieben die Interpretcn, drittens, fragwürdige empirische Behauptungen und historische Deutungen nach. Der mit dem Blick auf Weimar angeführte Hinweis auf die mangelnde politische Reife der Bevölkerung legt die polemische Frage nahe, ob sich denn die Staatselite in den zwanziger und drcißiger Jahren politisch als so reif erwiesen habe, daß zu ihren Gunsten der Grundsatz der Volkssouveränität einzuschränken wäre. Weniger polemisch, aber demokratictheoretisch wohl folgerichriger, ist zu fragen, wer oder welche instanz in einer demok racisch verfaßren Gesellschaft den einzelnen Gruppen und Individuen die politische Reifeprüfung abnehmen darf und wie sich in einer Demokrarie, die nicht als staatliche Veranstaltung verordnct und kontroliert wird, sondern ais kollektiver Lernprozeß institutionalisiert ist, die sicherlich wünschenswerte politische Reife der Bcvölkerung, d. h. ihre politische Konfliktfähigkeit, Handlungsbereitschatt und Toleranz erwerben läßr, wenn niche durch demokratische Prozesse. Solche Prozesse sind eigensinnig, in ihren Ergebnissen nichr immer absehbar und insofern nichr obne Risiko. Ein demokratisches Verständnis von Volkssouveränität läßı jedoch eine komplctte Risikoüberwälzung auf die Legislative, gar auf die Exekutive nicht zu.

Nicht von der Hand zu weisen ist, viertens, die Feststellung, plebiszitäre

33 Osscobühl, 3. a. O., 6 l. Vgl. auch D. Meren, Gedanken zur Demonstrationsifciheit, a. a. O., 621: - Dic im Grundgesezz verankertc repräsentative Demokratic (geht) von der Reprisentauon des Gesamivolkes (aus) und (lehnt) die Gruppenvertrerung (ab)*.

34 Womir nichs behaupter wird. Texinalie losc alle hermeneutischen Probleme. 
Betciligungsformen am Entscheidungsprozeß inplizierten eine Reihe von praktischen Schwierigkeiten. Weder gebieret diese Erkenntnis aber, den Grundsatz der Volkssouveränität als fundamentales Legitimationsprinzip einzuschränken, noch verwehrt sie uns, Demonstrationen als plebiszitär-demokratische Äußerungs- und Aktionsformen auszuzeichnen, etwa weil diese praktischen Schwierigkeiten gleichsam als immanente Grundrechisschranken vom Verfassungsgeber bedacht worden und deshalb bei der Verfassungsinterpretation zu berücksichtigen seien. Was als Argumenr gegen die verlassungsrechtliche Legirimität und gegen die Prakuikabilitär unmitrcibarer Demokratie gedacht ist, erwcist sich bei Lichte geschen, als versteckte Parlamentarismuskritik, die nicht bereit ist, die "Bctriebsrisiken " demokratischer Prozesse zu akzeptieren. Die propagandistische Formel von der "Unregierbarkeit* der Gesellschaft suggeriert im übrigen, daß das Verfassungsgebot, in repräsencativen Verfahren auf der Basis von Mehrheitsentscheidungen komplexe politische Probleme in einer kompromißkähigen Weise zu bewältigen, nicht mehr realitätsgerecht sei. Den $\nsim$ Unregierbarkeitstheoretikern» geht es angesichts der sich abzeichnenden fundamentalen Probleme der Systcmreproduktion um die Freiserzung rechnokratischer Imperative sozialer Sreuerung aus den Fesseln des demokratischen Prozesses", der für sic allenfalis die ornamentale Funkcion von Massenakklamacionen besitzt. Demgegenüber ist - gerade wenn man dic Schärfe dieser Krisendiagnose teilt und die Relevanz der alternativen policischen Lösungsmöglichkeiten durchaus ähnlich einschätzr - auf ciner strikten Bindung solcher Fundamentalcntscheidungen an den. Prozeß demokrarischer Selbsibestimmung zu insisticren. Nur auf diese Weise kann dem Demokratiegebor der Verfassung entsprochen werden. Wenn nämlich die Dimension der zu bearbeitenden gesellschaftliclien Problemc, wie Acomkraft, nukleare Aufrüstung und die Bewältigung ökonomisch-sozialer Konflikı, den Rahmen repräsentariver Verfahren sprengt, weil Kompromisse nicht zu erzielen sind und nicht nur die "laufenden Geschäfte" der Gesellschaft, sondern die Sicherung ciner Lebensform und des Überlebens der Gatcung Mensch auf der parlamentarisclien Tagesordnung stehen, und erst recht, wenn bereits die Wahrnehmung und öffentlichc Artikulation dieser Probleme an einer - das scheint im Hinblick auf die Exekutive noch euphemiscisch gesprochen - „Mauer der Ignoranz der Parteien und Parlamente scheitert, werden sowohl die Kapazität als auch die Legitimitätsschwelle des parlamentarisch-repräsentauven Systems übcrschritten. Es fragt sich, ob nicht in solchen sozialen Lebens- und Überlebensfragen von Verfassungsrang plebiszitäre Entschcidungsveriahren ebenso geboten sind wie bczüglich der grundlegenden Kanscitution, der Inkraftsetzung der Vcrfassung selbst. Keine Frage ist, daß Problemlagen, in denen politische Entscheidungsalternativen formell mehrheitstähig sein mögen, wegen der unabsehbaren Folgen aber kaum mehrlucitlich verancworter werden konnen, einen extensiven Schutz gerade der konstiturionellen Garantien gebieren, die die Souveränitär der Bürger und niche reglementiente öffentliche Auseinandersetzung verbürgen.

Bevor wir diese verfassungstheoretischen Überlegungen im Hinblick auf die Demonstrationsfreihcit konkrevisieren, ist eine besonders für dic deutsche Verfassungstradition nicht selbsstverständliche Implikation des Grundsatzes demokratischer Volkssouveränirät zu betonen: der Schurz politischer Minderheiten. Es ist bereits begrifflich das Schicksal jeder Opposition, noch nicht die Mehrheit zu besizzen. Aus der Unteilbarkcic der Volkssouveränitär folgt jedoch, daß die Legisımationswirkung auf der Statusgleichhcit aller Bürger beruht. Die Tatsache,

3s Vgl. hicrzu C. Offe, "Unregierbarkeita. Zur Renaissance konservativer Krisentheorien, in: J. Habermas (Hg.), Stichworte zur , Geistigen Situation der Zeit, Bd. I (Frankfur, 1979), 194 ff. 
daß bescrimmte Auffassungen oder Interessen herrschend oder mehrheitsfähig sind, verschafft diesen keine höhere Legitimität, wenn auch qua Mehrheitsprinzip mehr Wirksamkeit im politischen Entscheidungsprozeß. Eben deshalb will das Grundgesetz mic seinen Diskriminierungsverboten (Art. 3 III, 33 III) sicherstellen, daß minoritäre, $d . h$. politisch unterrepräsencierte Positionen weder vorab noch nachträglich aus dem politischen Prozeß ausgeschaltet und ihre Prougonisten rechtlich ausgebürgert werden. Die Statusgleichheit aller Bürger ist besonders dann in Gefahr, rvenn die Staatsgewalt, wic vom Grundgesetz vorgesehen, durch repräsentarive Organe ausgeübc und allgemein verbindliche Entscheidungen nach dem Mehrheitsprinzip gefällt werden. Diescr Gefahr beuge das Grundgeserz mit einer Reihe von Sicherungen gegen eine Tyrannei der (parlamentarisch dominierenden, also nicht notwendigerweise zahlenmäßigen) Mehrheit vor. Die policischen Entscheidungsverfahren sind gewaltenteilig strukturiert und ihre Resultace der Justizkontrolle unterworfen (Art. 20 III, I9 IV, 92 und 97 GG). Die an die politischen Gewalten delegierten Komperenzen sind formell cingeschränkt, wic erwa durch das Zitiergebot oder das Verbor, Einzelfallregelungen zu treffen oder die Verfassung zu durchbrechen. ${ }^{j 6}$ Bestimmte Gegenstände, vor allem dic Fundamentalprinzipien des demokratischen Entscheidungsprozesses selbst (Art. 79 III), die durch dic Wesensgehaltsgarantie (Ar. 19 II) hinsichtlich des Grundrechtsschurzes noch verstärks werden, stehen nicht zur Disposition der gewählten Repräsentanten. Ebenso wie diese verlassungsrechtlichen Kautelen läßt sich auch die Institurionalisierung ciner Verlassungsgerichrsbarkeit als Versuch interpretieren, dem Schutz politischer Minderheiten Geltung zu verschaffen. ${ }^{\prime 7}$

Den unbequemen Konsequenzen eines strikten Schuczes polirischer Opposition und damit der prakrischen Virulenz politischer, sozialer wie kultureller Alremariven im Rahmen der genannten Einschränkungen der Arr. 79 III und 19 II GG - haben sich dic mannigfachen Verlassungsinterpreten immer wieder zu entziehen versucht. Unter Berufung auf die Konzeption einer sstreitbaren Demokrarie « sind Diskriminierungsserategien der Exekucive auch vom BVerfG legitimiert worden. ${ }^{8}$ Uncer Berufung auf vordemokratisclie Traditionsbestände (nhergebraclitc Grundsäıze“, „besondere Gewaltverhältnissea), auf eine überpositive "Wertordnung , auf cinen vermeintlichen Grundkonsens ("Keine Freiheir für dic Feinde der Freiheit ") oder neuerdings auch auf die Funktionsimperacive des politischen Systems und seiner Teilbereiche" haben die politischen Gewalten den Schurz politischer Minderheiten relativier oder leerlaufen lassen und die politischen Freiheitsverbürgungen, auf deren Wirksamkeic gerade Oppositionsbewegungen angewiesen sind, für die gesamte Sphäre der expandierenden Staatstätigkeic (so u. a. im ölfentlichen Dienst, in der Bundeswehr in den Schulen) parriell eingezogen. Solche diskriminierende Staatspraxis hat zugleich das vorkonstitutionelle Vernunftpostulat kompromirticrt, das Grundlage und logische Voraussetzung der verfassungsmäßigen Verankerung von Demokratie ist: $D_{2} ß$ nämlich allen Bürgern uncerschiedslos und in gleicher Weise die Fähigkeit zu racionaler Einsicht und autonomer Encscheidung zukommt, durch dic cin vernünfig angeleiteter Wille der politischen Gewalten überhaupt crst konsticuiert - und vor allem legicimicrt werden kann.

Das Grundgeserz ist daher über die auf W. Abendroth zurückgehende politisch-so-

$36 \mathrm{H}$. Ehmike, Verlassungsïnderung und Vcrissungsdurchbrechung, AöR 79 (1953/43), 396 if.

37 Zum Mandat der Verfassungsgerichtsbarkeit, politische Minderheitcn zu schuezen, vgl. G. Franken-

berg/U. Rödel, Von der Volkssouveranität zum Minderheirenschutz. Dic Frciheil poltuscher Kommunikation in Verfassungsstant (Frankfurt, 19\$1), 3 jo f.

$3^{8}$ Vor altem im *Radikalenbeschluß". BVerfGE 39. 334 ff.

$39 V_{g l}$ dazu E. Ricble, Funkcionseichtige Stratrechtspncge, $\mathrm{KJ}{ }_{3} / 80,316$ ff. 
ziologische Bestimmung als gesellschaftlicher Kompromiß oder als "Waffenstillstanósabkommen * der antagonistischen sozialen Kräfre hinaus auch in der normativen Dimension politischer Moral als Verpflichtung aller Bürger zu interpretieren, im gesellschaf́lichen Verkehr Auronomie, Integrität und Gleichheir ${ }^{\circ}$ wechselseitig zu respektieren. Das Legirimationsprinzip politischer Herrschaft, der Grundsatz der Volkssouveränität, crstreckt diesc Verpflichtung aúf die Scaatsgewalt. Das von dieser reklamierte, den demokratischen Konstitutionsprozeß und sein dialogisches Prinzip potentiell sprengende "Monopol legitimer Gewaltsamkeit a gebietet besondere Vorkehrungen, um der strukturell drohenden obrigkeitlichen Verletzung von Auronomie und Glcichheit vorzubeugen. Die krisenhaften Akkumulationszyklen der Ökonomie, die Interessengegensätze einer antagonistischen Gesellschaft und die versachlichten System\%wänge der staatlichen Herrschaftsagenturen legen es nahe, nur sehr begrenzt auf die soziale wie policische Wirksamkeic subjektiv sinnhafter, nicht instrumentalistisch verkürzter Standards kollekriver wie individueller humaner Lebensperspektiven zu vertrauen. Freiheitsverbürgungen, wie Streik- und Demonstrationsrecht, und Diskriminierungsverbote, einerseits, Gewaltenteilung und die Institutionalisicrung politischer Mehrheitsentscheidungen andererseits lassen sich als ein kompromißhafter Versuch auffassen, Autonomie und Gleichheit aller Bürger in einer rechtlich gesicherten, prakrikablen und pragmatischen Weise zu realisieren: -Prakcikabel, weil es sich in einem Flächenstaal und wegen der konsensuell nicht zu lösenden Konflikte anbiezet, diese Postulate nicht in der ihnen an sich angemesscnen Form einer direkten Demokratie zu insticutionalisieren, in der Entscheidungen einmütig getroffen werden müßten, sondern den politischen Enrscheidungsprozeß durch Repräsentarion und nach Maßgabe des Mehrheitsprinzips zu vereinfachen. Der Pragmatismus äußert sich in den Vorkelirungen fur die justizielle Beilegung von Konfliktcn durch eine - dem Anspruch nach - neutrale und unparteiliche Instanz ... Dementsprechend verkörpert die Verfassungsgerichtsbarkeir die (begründete) Sorge politischer Minderheicen, die Majoritär, $d$. l. die das repräsentaciv-parlamentarische System dominierenden Interessenkoalitionen, könnte die sgrundlegende Konvention, (covenant) der Gescllschaft brcchen, das Gesetz selbst in die Hand nehmen oder sich unter Einsarz des statlichen Gewaltmonopols einer ihr unbequemen Opposition entledigen. $\alpha^{4 t}$

In diesem verfassungstheoretischen Kontext kommt der Freiheit politischer Kommunikation, mithin auch der Demonstrationsfreiheit als verfassungsrechtlicher Verbürgung und Ermöglichung polizischer Selbsträrigkeit der souveränen Bürger und als Schutz politischer Minderheiten eıne hervorragende Bedeutung zu. Demzufolge darl die Entscheidung über die Zulässigkeit oder Unzulässigkeit von alternativen Formen demokratischer Willensbildung und über die Chancen von Minderheiren, ihre Inreressen zu artikulieren, zu organisicren und politisch durchzusetzen, nicht den parlamentarischen Mehrhcicen überlassen bleiben. Diese grundgesetzlich gesicherte Möglichkeit, die nichr repräsentativ-parlamentarisch vermitrelte politische Aktivität der Bürger offen zu halten, ist die logische Konsequenz demokratisch-souveräner Selbstregierung. Der Kampf dafür ist immer auch Thema jeder Demonstracion.

40 Gemeint ist hier eın ziviler Begriff von Wurde, die gerade In der Anerkennung der Einsichtsfahigkeit und Emöglichung autonomer Entscheidungen des Individuums zum Ausdruck kommt. Vgl. R. Dworkin, Taking Rights Seriously (London, 1977), 198 ff.

$4^{1}$ Frankenberg/Rödel, a. a. O. 
Kollektivc polirische Aktionen in der Öffentlichkeit beeinträchtigen allemal dic Büngerruhe; kommt es im Zusammenhang mir solchen Akrionen auch nur zu vercinzelten gewalträtigen Übergriffen, so ist die bürgerliche Ordnung gcstört. Nicht nur solche Übergriffe zu ahnden, sondern möglichst gleich dic kollektive Inanspruchnahme politischer Freiheitsgarantien einzuschränken, wenn nicht gar zu verhindern, ist, wie dic Geschichte des Demonstrationsrechts zeigt, verbreitete Staatspraxis, die auf ein ausdifferenziertes straf- und zivilrechtliches, verwaltungsund polizeirechtliches Instrumentarium zurückgreifen kann. Um den konstiturione!l gebotenen Schutz politisch-oppositioneller Gruppen, ihrer Programmatik und Arrikulacionsformen zu gewährleisten, sind daher die bisher angestelltren verfassungscheoretischen und grundrechtsdogmatischon Enwägungen wenigstens ansatzweisc zu konkretisieren und Grundsärzc für einen „Demokratietest « der exekutiven, judikariven und Icgislativen Reglementierung des Demonstrationsrechts zu skizzieren. ${ }^{\text {t2 }}$

(1) Jede Bervertung und recheliche Diskriminicrung der Inbalte politischen Protests ist unzulässig. Denn zum cinen stchen objektive Kritericn und Maßstäbe für die Bcurteilung der sozjalen wie politischen Nüzzlichkeit einzelner Beiträge zur öffentlichen Diskussion niche zur Verfügung. Zum anderen würde die Einführung solcher Bewertungsstandards durch parlamentarische Mehrheiten die Demonstracionsfreiheit ihrer kritisch-aufklärerischen Stoßrichtung und ihrer Polemik gegen den selbscherrlichen Immobilismus des etablierten politischen Institutionensysterns und seiner öffentlichen Selbsıdarscellung berauben. Es ist daher unzulässig, ein einheitliches Ordnungsbild legislariv zu fixieren und polizcilich-justizicll zu garantieren", auf dessen Folie bestimmce inhaitliche Forderungen oder Kritik oppositioneller Gruppen für ordnungswidrig, für ungeeignet zur politischen Willensbildung oder für staztsabrräglich erklärt werden. Die Legitimität und Schutzwürdigkeit von Demonstrationen kann auch nicht von einem spezifischen politischen Bezug abhängig gernacht werden. Dieser ergibr sich bereits aus der Öffentlichkeit des Protests und der sich darin manifesticrenden Absicht, auf die Mirdemonstrierenden, auf das Publikum der Andersdenkenden oder dic politischen Getvalten einzuwirken" ${ }^{4}$ oder ganz allgemein auf soziale Mißstände aufmerksam zu machen. Es ist nicht Sache der Staarsgewalt, über den Sinn und Zweck, den Werr und die Vernünfrigkeit

42 Die im folgenden entwickelten Grundsärze geleen ebenso für das Demonstrationsstrafreche wie fur die in den letzeten Jahren immer häufiger verílgie Strarcgic, dic Demonsurationsschäden und auch die Kosten des Einsatzes der Staatsgewalt bei den Demonstranten zu liquidieren - damit also cinmal die Inanspruchnahme politischer Freiheitsgarantien mit einem kaum ubscrschaubaren Hafungsrisiko zu belasien und den regelmäßigen Vollzug der Staatstäeigkeil als Privatlcistung zu monetarisicren. Zum Vorrang polusscher Aufklärung und Kommunikation gegenuber Geld- und Sachschäden vgl. R. Wwetholect, Zur polusechin Funkcion des Rechus am cingerichtecen und xusgeubten Getverbebetrieb, in: KJ $2 / 70,121 \mathrm{ff}$.

43 Vgl. auch Denninger. Polizei in der freihcitlichen Demokratie, a. a. O., 32. Zur rechtichen Gleichgültigken von Demonstrationsziclen vgl. Baumann/Frosch, Der Entwurf des 3. Strafrechtste-

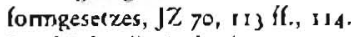

44 Je schärier die Aniorderungen an einen (sichtbaren) polikischen Bezug gestell werden, umso großer ist die Gefahr, daß sich cin herrschendes Politikverständnis auf den, Wege der Intcrpretation als Schranke der Demonstrationsfreiheit auswirkt. Vgl. Denninger, ZRP 68, 42, der lordert, den politischen Bezug weit zu lasscn. Für Herzog. MDH. Arr. 8, RdNr. 43 isı die Frage, ob für einc politische Angelogenheit demonstriert wird oder nacht, nicht mit der Funktion des Ar. S GC vercinbar, die Isolierung des Individuums aufxuheben. Im ubrigen erscheint es absurd, auf den besonderen politischen Charakter öf(erelicher Akcionen abxuseellen, wenn andererseies das Privateste, nämlich das Gevissen (der wehtfähigen Männcr) zum Polieikum gemache wird. 
von Demonstrationen zu befinden. Denn sie könnte versucht sein - und isz es oft genug - zu tun, was Demonstrationsfreiheit gerade verhindern will, nämlich mißlicbige Kritik und Aufklärung zu unterbinden. ${ }^{\text {st }}$

Rechcliche Sankrionen im Bereich politischer Auscinanderserzungen sind nur sorwcit legitim, wie sie entsprechend dem Schurz parlamentarischer Redefreiheit (Art. ${ }_{4} 6$ Abs. I GG) als verlcumderische Beleidigungen die Autonomic, Würde und Gleichhcit der Bürger mißachten. Wer beispielsweise meint, politische Kritik mit rufschädigenden Äußerungen über Mitbürger munitionieren zu müssen (über die Grenzen eines Rechrs zum polemischen, Gegenschlag hinaus), irägr das Risiko einer gerichtlichen Auseinanderseczung." Eine analoge Behandlung von Kricik, die sich gegen staatiche Insticutionen richtct, scheider jedoch aus. Denn dic Staatsgewalt kann sich nicht gegenüber dem souveränen Burger auf die „Würde " von Institutionen oder Symbolen berufen und strafrechtlichen Ehrenschutz einklagen, weil der Staar als Rechtsperson die Insignien personaler Integritäı nicht besitzt. ${ }^{46}$ Der strafrechtliche Ehrenschurz des Stantes, etwa das konturenlose Delikt der "Verunglimpfung des Staates und seiner Symbole ( $\$$ goa $\$ r G B)$, eröflnet in diesem Sinn nicht nur ein Einfallstor für dic inhalıliche Zensur policischer Auscinandersetzungen, sondern mache den Staat auf diese Weise selbst zum Ferisch, der sich hinter einer Tabuzone der Kritik, einer Bannmeile des Protests, verschanzt.

(2) Das konsticutionelle System der Bundesrepublik verleiht weder einem einzelnen Aspekr, wie erwa der verbalen Äußcrung"7, noch einer spezifischen Form politischen Protests exklusive Legitimicät. Auch die parlamentarische Opposition kann für sich gegenüber der außerparlamentarischen keinen Vorrang oder einen Legalitätsbonus reklamieren. ${ }^{48}$ Es ist grundsäczlich Sache der Becroffenen zu entscheiden, in welcher Form sie ihr Anliegen publik machen oder ihre Kritik demonsrrieren wollen. Die staatliche Lizenzierung von Protestormen ist mit dem Demokratieprinzip unvereinbar, da sie den Grundsarz der Selbscbescimmung aufgibe und die Wirkungschancen oppositioneller Kräfre in das Ermessen der Legislative oder Exekutive steilt. Das Verständnis des Demonstrationsrechts als einer wesentlichen Voraussetzung für den Schutz politischer Minderheiten erlegt der Staatsgewalt Verpflichtungen auf, die über das Verbot prinzipieller Versammlungsfeindlichkeit ${ }^{49}$ hinausgehen. Auch ein bloßes Willkürverbot bei der Vergabe von Versammlungsräumen oder bei Auflagen für Demonstrationen sichert politischen Minderheiten niche ausreichend die Möglichkcit, Mehrheir zu werden" Vielmehr können sie sich auf ein positives Statusrecht berufen, das ihnen den Zugang zu öffentlichen Foren für die Arrikularion ihres Protestes sicheri', den die Staatsgewalt nicht nur zu tolerieren, sondern zu

442 Daß ein Parlament, wic der Bundestug vor der Friedensdemonstration vom 10. 10.1981 in Bonn, hergeht und über die zu erwanenden politischen Aussagen der Demonsteationsredner, über die mutmaßlichen Morive der Demonsimutonsteilnehmes und uber die Norwendigkeit der angekündigten Demonstration debatticre und cneschließt, offerbart ein groceskes Mißverstindnis der Funkuion und begrenzten Legitimation repràsentauv-parlamentanscher Organc einerseits und der Bedeutung polizischer Freiheitsrechte in einer Demokratie andererseits: vg. BT-Dnucks. $9 / 871$ und g/87/4, 57 . Sitzung, 9. 10. 1981, S. 3311 ff.

4s Vgl. Frankenberg/Rödel, a. a. O., 332.

${ }_{4} 6 \mathrm{D}$. Sterzel, Nur der autoritare Stan verfolga Kritik, in: Th. Blanke u. a., Des Oldenburger Buback-Prozeß, Berlin 1979, S. 203 If. Im amerikanischen Verfassungsrecht wird deshalb die Kritik an politischen Amsträgern auch tendenziall privilegien; vgl. Frankenberz/Rodel. 1. ง. O., 196 if.

47 An. s I schutze - beispielbaft - Meinungsaußcrungen win Worn, Schrift und Bild * Und die Information waus allgemein zuganglichen Quellena. Wie hier auch v. Münch, GG, Ar. s, RdNr. p.

48 BVerfGE 20, 56/97 ff.

49 So MDH, Arr. 8. RdNr. 21, Dis OVG Lincburg hält den Stast für wweingehend verpflichret, die Abhalung von Versammlungen unter freiem Himmel zu ermöglichen a (NJW 78, 1939).

50 $\mathrm{MDH}, \mathrm{Ar} .8, \mathrm{RdNr}$. 21; Dietel-Ginıel, $\{1,17$, spricht von einem "Differenzienungsverbot $\alpha$

s1 Dis überwiegende Lehre und Kspr. lehnt eine solche Inserpreration ab, s. o. Anm. 43 und 44 sowic 
schützen und zu ermöglichen hat. Genuiner Sinn der Freiheit politischer Kommunikation ist, daß soziale Gruppen, deren interessen systematisch oder punktuell unberücksichtigı bleiben, eine möglichst wirksame Gegenöffentlichkeit endalten können. Um Aufmerksankeit zu erregen und politische Resonanz angesichts einer verfestigten öffentlichen Meinung zu erzielen, wird verfassungsrechtlich garantiert, daß Protest öffentlich inszenicrt und dramatisiert werden kann. Politische Opposition und Minderheiten bedürfen folglich eines verfassungsrechtlich garantierten Zugangs zur Öffentlichkeit.

"Friedlichkeit" und "Gewalttätigkeit " markieren die Grenze legalen und damit im vorherrschenden Verständnis legirimen und illegalen, daher illegitimen Protests, wie auch Art. 8 I GG nur friedliche Versammlungen schützc. Mir dem Gewaltverbor allein ist freilich eine eindeurige Grenze nicht gezogen, weil, wie die Analyse der einschlägigen Rechtsprechung gezcigt hat, interpretativ umstricten ist, welche Procestformen als gewalaätig anzusehon sind und welche nicht. Je nach verfassungstheoretischer Konzeption und politischem Standort der Verfassungsinterpreten ändert sich der Grenzverlauf: In einer am staaclichen Ordnungs- und Sirafanspruch orientierten Judikatur kann bereits die bloß̉e Präsenz in einer auch nur beiläufig wunfriedlichen" oder sogar bloß potenciell militanten Protestaktion strafrechtlich relevant werden. Anderen Interpreten erscheint dagegen auch die gezielse vorübergehende - Blockade publizistischer Geschäftstätigkeir oder öffentlicher Verkehrsmittel noch demokrarieadäquat. ${ }^{2}$ Sicht man von den klaren Fällen wie etwa Körperverletzung, Brandstifrung oder Werfen von Steinen und Sprengsärzen ab, so besteht hinsichclich der Einschätzung des verfassungsrechtlichen Schuizes und des Unrechtsgehalts aller unter dieser Schwelle von Gevalt liegenden Protestformen. wie z. B. Sitzstreik, Wegdrängen van Polizeiketten"s, Werfen von faulen Eiern und Farbbeuteln, Schaukeln eines PKW, Dissens. " Ohnc diescn hier auflösen zu wollen, halten wir doch für die Grenzziehung zwei Gesichtspunkte für wesendich.

Erstcns - und das bleibt in der Gewalcdiskussion weitgehend unberücksichtigt gelten verschiedene Formen der Beeinträchtigung der physischen und psychischen Integrität außerbalb des Kontextes politischer Auseinandersetzungen durchaus als in Kauf zu nehmendes Risiko sozialer Kontakte. Daß Volksfeste oder Sportveranstalrungen, daß Massenveranstaltungen generell nicht ohne Konflikte abgehen, die zum Teil auch gewaltförmig ausgetragen werden, hat noch niemanden moriviert, allen Ernstes ihr Verbot oder ihre strike staatliche Kontrolle zu fordern. Im Straßenverkehr bewegen wir uns unter den Bedingungen »mitwirkender Betriebsgcfahren and offenbar sozialtypischer, jedenfalls weichin akzeptierter Formen von Gewalt. Bei populären Sportarten wie Fußball und Boxen (von Aurorennen ganz zu schweigen) wird ein beachtiches Maß an Körperverletzungen als "Kunst der Selbstverteidigung* oder ncnglische Härte* nicht nur hingenommen, sondern als prolessionclles Verhalten gewürdigt. Erst der spezifisch politische Kontext provozicrt die Staatsge-

Nachrw, bei Dietel-Gintzel, a. a. O. Vgl. auch Meren, Demonstrationen umecr dem Gesetz, a. 2. O., 60 und Frowein, NJW 69, 1084 . In der Ablehnung des status positivus schlägt regelnäßig die Vorstellung durch, Demonstrationen scien *Sondemutzung * und nicht Gemeingebmuch der öffentlichen Verkehrswege und Plätze. Dic amerikanische Verfassungslehre und die Rechtsprechung des Supreme Coun haben - wenn auch nicht uncingeschränkt - den Anspruch politisch-oppositioneller Gruppen auf ein "public forum" anerkannt; vgl. H. Kalver, The Concept of the Public Forum: Cox v. Louisiana, in: Supreme Coure Revicw (1965), i ff.

S2 Z. B. R. Wietholter, a. 2. O., Is ff.

S3 BGHSt 23, ss und wohl auch v. Bubnoff, Leipziger Kommentar zum SiGB (LR), 10. Aufl, \$ 125, 2s: a. A. OLG Fim, KJ H. 1/70 S. s9 f

34 Nachw bei v. Bubnoff、 LK, \$125, 25; Schönke-Schröder, Strafgeseizbuch, 20. Aufl. (Munchen, 1980). \$129, 6 if. 
walt zum Einsacz ibrer Strategien möglichst präventiver Gewaltverhinderung, dic sich implizit gegen die Artikulationsfreiheir politischer Opposition richten.

Zweitens ist zu bedenken, daß Demokratie und Volkssouveränirät die Bürger nicht als Objekte staatlicher Maßnahmen oder "Staatsdiener" und auch nichr nur als "Staatstheilnehmer oder Publikum ins Auge fassen, sondern als autonome Subjekce ihrer gesellschaftlichen Angelegenheiten und ihrer politischen Geschäfte. Arr. 201 und II , GG verbürgt ihnen das Recht und uncerstellt ihnen die Kompetenz zu demokratischer Selbstregierung. Das bedeucet, daß sie bei rechelicher Ancrkennung und rechrlichem Schutz ihrer Privarsphäre als souveräne Subjekte des politischen Gemeinwesens politische Auseinanderseczungen tolerieren und auch deren Risiken - wenn auch nicht unbegrenzt - hinnehmen müssen. Eine so verstandene * Politikpflichtigkeit vorlangt ihnen keine eigene politische Aktivität ab. Wohl aber begrenzi sie den polizei- und strafrechclichen Schurz eines möglichen Bedürfnisses, in Ruhe gelassen, mit politischon Fragen nicht behelligi und vor jedweder Belästigung etwa durch Demonstranten bewahrt zu werden." Wcr mangels Neigung oder Übung kontroverse policische Auscinanderserzungen in der Öffentlichkeit verabscheur und jede kollekrive Akrion, weil es zu Ausschreitungen kommen könnte, ais bedrohlich empfindet, kann von einer demokratisch verpflichtcten und rechtsstaatlich gezügelten Staatsgewalt nicht verlangen, ihm dieses Bedrohtheitsgefühl zu ersparen. Nicht politischcr Quictismus, sondern Freiheit von Angst ist zu schürzen; und zwar die Angst vor konkreren Bedrohungen, die den demokratischen Rahmen sprengen. ${ }^{16}$ Die cinschlägigen Bestimmungen des Demonstrationsrechts, ihre administracive Handhabung und die dazu ergangenen gerichclichen Entscheidungen sind daher stets daraufhin zu überprüfen, ob und inwiewcit sie den notwendigen und von der Verfassung gebotenen Schutz von Individualrechtsgütern leisten - also Leib, Leben, Gesundheit, persönliche Freiheit und persönliches, d. h. autonomieverbürgendes Eigentum schützen. Oder ob sie über den vorgeblichen Schulz eines diffusen allgemeinen Rechtssichcrbeitsgefühls ${ }^{77}$ oder eines vagen nöffenclichen Friedens " ${ }^{38}$ demokratische Prozesse domestizieren und entpolitisieren, um die Autorität eines sich verselbständigenden staatlichen Hoheitsanspruches gegen leginime Ausdrucksformen der Bürgersouveränität durchzusetzen.

(3) Was die Verfassung verbürgr, kann der Gesetzgeber nicht verbieten. Damit ist niche naiv behauptet, daß alle konstiturionellen Garantien als uneinschränkbare verbrieft sind, sondern daß gerade die Äußerungs- und Aktionsformen, die wegen ihrer konstituriven Bedeutung für demokratische Prozesse besonderen Schurz genießen, vom Staat nicht diskriminier werden dürfen. Sinn der Demonstracionsfreiheit im Kontext des demokratischen Prozesses ist es, offentliche, kollektive, außerinstitutionelle und dramatisierte, also nicht rein verbale Äußerungen und Aktionen zu ermöglichen. Diese Wesensmerkmale von Demonstrationen sind dem parlamentarischen Gescrzgeber wie der Exekurive als Anknüplungspunkte für Sanktionen oder Reglementierungen entzogen. Verfassungswidrig sind deshalb beispielsweisc Straftatbestände, die - um der öffentlichen Sicherheit und Ordnung, um des öffentlichen Friedens oder um der Bürgerruhe willen - darauf abzielen. öffentlichen Protest als Friedensbruch, kollektive politische Aktionen als (abstrakte)

ss Vgl. dazu Baumann/Frosch, a. a. O., H. Simon, Freihcitichc Verlassung und Demonstravionsreche. Berlincr Reden Nr. 17. 1969, 12 u. 19 und auch R. Wietholecr, 1. 2. O.

s6 Vgl. G. Frankenberg, "Landfrieden und Demonstrationsfrciheit, KJ $3 / 80,260$ ) ff.

57 So das Schutzgut des $\$ 12$, StGB gem. der Kommentierung von Schönke-Schröder, $\$ 12\}$,$\} .$

¡8 Nahezu alle sog. Massendelikee sollen auch, wenn nichu primär, den ölfentlichen Frieden, die offentliche Sicherhcit und/oder dic äfentliche Ordnung schürzen; vgl. die Kommentierung und die Rspr. Nachw. bei v. Bubnoff und Schonke-Schröder zu den $\$ 124,129,1252,126$ S2GB. 
Gefahr für die Rechrssicherheit, staatlich nichr kontrollierte Opposition als

Bedrohung der Rechtsordnung und niche maßvoll geäußerre Kritik an der Staatsgewalt als "Verunglimpfung" zu illegalisieren." So etwa die alte Fassung des Landfriedensbruchtatbestandes, der bereits den Anschiuß an eine Menge, die bloße Teilnahme an einer »Zusammenrottung* unabliängig von der friedlichen Absicht und des konkreten Verhaltens pönalisiertc ${ }^{60}$; oder die Konstruktion einer "psychischen Mittäterschaft a friedlicher Demonstrancen an vereinzelten Ausschreitungen ${ }^{61}$; oder der Verwcis auf eine "Erfahrung, die das Mißtrauen der Staatsmacht gegen eine zu einem nicht erkennbar-harmlosen Zweck zusammenströmende Menschenmenge" begründen und sogenannte Massendelikte rechtínertigen soll. ${ }^{62}$

Will man demgegenüber verhindern, daß das Demonstrationsrecht die Grenzen des gebotenen Rechrsgüterschutzes in eine antidemokratische Richtung weiter überschreitet, so muß das grundgesetzliche Primat der Freiheir politischer Betatigung ernst genommen werden. ${ }^{6}$ Die fundamentalen Organisationsprinzipien einer demokratisch verfaßten Gesellschaft: Volkssouveräniät, Recht auf policische Opposition und Schurz politischer Minderheiten, führen für das Demonstrationsrecht zu der Regel, daß geseczliche Regelungen und administracive Maßnahmen, die die Freiheit zu demonstrieren cinschränken, unter einem besonderen Begründungs- und Rechtfertigungszwang hinsichtlich ihrer Demokratieadäquanz stehen.

s9 Wie liver auch MDH, Art. 8, RdNr. $82 \mathrm{~m}$. w. Nachw, OU, NJW 69, iss und Tiedemann, Bemcrkungen zur Rechisprechung zu den Demonstratsonspnozessen, JZ 69, 71) $11 ., 719$.

6o Vgl. v. Bubnoff, LK, Vorbem. ru $\ 12$ j ff.

61 Schönke-Schroder, $S 125,88$ laßr nur psychische Ansuifung und Beihilfe zu. Weirergehend v. Bubnoff. \$125. 5, 7. Die Konstrukcion viner apsyshischen Mitizersehafu war Grundlage der Nurnberger Massenverhafiungen vom s. und 6. März 1981 und Kern der Anklageschriften geger dic muimaßlichen Tcilnchmer des Demonstration, denen keine eigenlı̈̈ndig begangenen Steinwürfe o. ä. und auch keinc konkreten Unterstülzungshandlungen nachgewiesen und zur Last gelege wurden. Vyl. dazu Frankenberg, 2. a. O.

62 Aus dur Stellungnahme des Verfassungsrechtsleheers Frowesn während der Hearings zum 3. Strafrechesinderungsgescizes, zit. nach $v$. Bulsnoff, Vorbem. 7u $\$ 125$ ff.. 2.

6) Eine solche Freiheitsvennuming ourde zugleicl zum Ausdruck bringen, daß Demonsirationen prinzipiell als Außcrungstormen nicht delegicrer Souveranitar der Burger gelten mussen, und daß dic Demonstrationsfreihcit deshalb nicht nut dic Funktion hat, dic "äußersten Xonsequenzen des Parteienstaates zu verhindem* (Ou, Gestz uber Versammlungen..., Einl. RóNr. s) oder die parlamentarischen Berstungen durch den öffentlich geäuBerten Volkswillen oder Volksunwillen aufuhellen. 